\title{
Ion implanted dielectric elastomer circuits
}

\author{
Benjamin M. O'Brien • Samuel Rosset . \\ Iain A. Anderson • Herbert R. Shea
}

Received: 14 June 2012 / Accepted: 9 October 2012

(C) Springer-Verlag Berlin Heidelberg 2012

\begin{abstract}
Starfish and octopuses control their infinite degreeof-freedom arms with panache-capabilities typical of nature where the distribution of reflex-like intelligence throughout soft muscular networks greatly outperforms anything hard, heavy, and man-made. Dielectric elastomer actuators show great promise for soft artificial muscle networks. One way to make them smart is with piezo-resistive Dielectric Elastomer Switches (DES) that can be combined with artificial muscles to create arbitrary digital logic circuits. Unfortunately there are currently no reliable materials or fabrication process. Thus devices typically fail within a few thousand cycles.

As a first step in the search for better materials we present a preliminary exploration of piezo-resistors made with filtered cathodic vacuum arc metal ion implantation. DES were formed on polydimethylsiloxane silicone membranes out of ion implanted gold nano-clusters. We propose that there are four distinct regimes (high dose, above percolation, on percolation, low dose) in which gold ion implanted piezo-resistors can operate and present experimental results on implanted piezo-resistors switching high voltages as well as a simple artificial muscle inverter. While gold ion implanted DES are limited by high hysteresis and low sensitivity, they already show promise for a range of applications
\end{abstract}

B.M. O’Brien $(\bowtie) \cdot$ I.A. Anderson

Biomimetics Lab, Auckland Bioengineering Institute, University of Auckland, Auckland, New Zealand

e-mail: ben.obrien@auckland.ac.nz

S. Rosset · H.R. Shea

Microsystems for Space Technologies Lab, École Polytechnique

Fédérale de Lausanne, Neuchâtel, Switzerland

I.A. Anderson

School of Engineering Science, Faculty of Engineering,

University of Auckland, Auckland, New Zealand including hysteretic oscillators and soft generators. With improvements to implanter process control the promise of artificial muscle circuitry for soft smart actuator networks could become a reality.

\section{Introduction}

A hallmark of life is the use of soft, flexible muscles to manipulate an organism's surroundings. Soft muscles are intrinsically compatible with unstructured environments so they do not require tight tolerances or high speed control, yet provide robustness under variable and uncertain conditions [1]. This is a robustness not shared by engineered systems based on hard and rigid technology such as electromagnetic, piezoelectric, or hydraulic actuators. The recent development of artificial muscle technology-Dielectric Elastomer Actuator(s) (DEA) (Fig. 1) [2]—provides an opportunity to build soft and truly lifelike robotic, mechatronic, and prosthetic devices. DEA are well suited to smart actuator networks [3] as they are capable of strong, silent, and large actuation [4], have already been incorporated into a wide range of biomimetic devices [5-8], and are made of cheap printable materials [9].

However, the provision of soft actuation creates a big problem in control. Soft actuator structures and networks can have many degrees of freedom that overwhelm a central processor $[1,10]$. Nature solves this problem by distributing control to provide speed, robustness, and computational simplicity. Consider two examples. Octopuses control infinite degree-of-freedom manipulators using characteristic behaviors mediated by neurons distributed throughout their arms-substantially reducing demands on the animal's brain [11, 12]. Starfish (see Fig. 2) have a distributed brain capable of complex behavior such as flipping the 

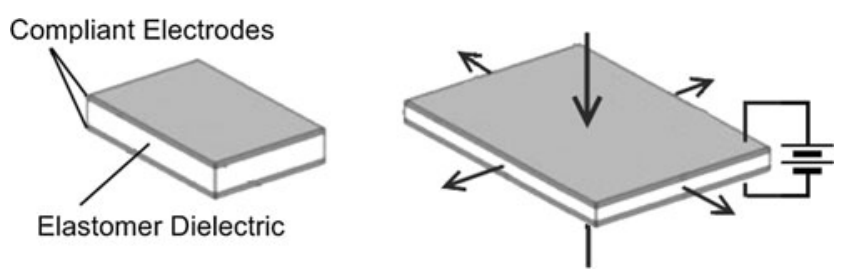

Fig. 1 Dielectric elastomers are soft flexible capacitors. When a high voltage is applied, electrostatic interactions give rise to an expansion in plane and compression in thickness. Image adapted from Gisby et al. [13]

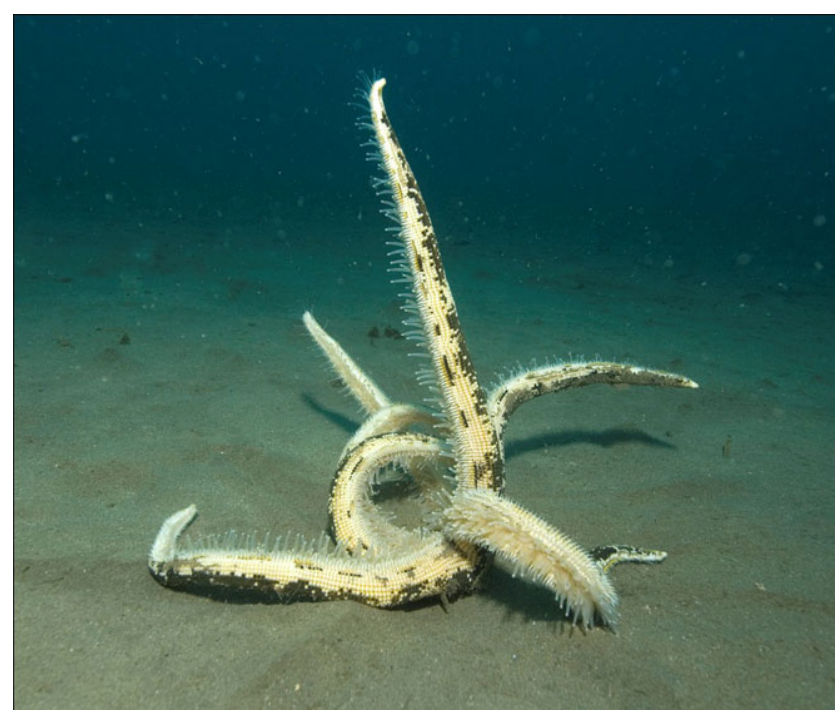

Fig. 2 Starfish are amazing examples of the soft, smart muscular networks seen throughout nature

right way up [14] whilst at the same time being incredibly robust-some starfish can reproduce by splitting in two $[15,16]$.

Clearly it is desirable to make use of this distributed control approach for artificial muscle networks. Unfortunately, however, conventional electronics are poorly suited to this task - they are rigid, hard, and brittle, meaning they impede movement or break when incorporated into soft structures. These limitations are well recognized by a rapidly growing stretchable electronics community, who are employing microfabrication techniques to make stretchable semiconductor devices [17-19]. Stretchable semiconductor electronics show promise; however, they make use of dissimilar fabrication processes to artificial muscles-complicating manufacture and increasing cost-and cannot be easily used to control high voltages.

Recently we presented the Dielectric Elastomer Switch (DES) [20] that can overcome these difficulties and allows for distribution of reflexes throughout an artificial muscle device. DES are piezo-resistive elements that can be patterned onto artificial muscle membranes to create soft electric circuits. Piezo-resistivity is the change in a material's re- sistivity with stretch. Piezo-resistive polymers are typically realized via the distribution of conducting particles such as carbon [21] or nickel [22] throughout an insulating polymer matrix. Piezo-resistive behavior originates from geometric effects such as the forming and breaking of conductive networks under strain, or from conduction effects such as quantum tunneling and electron hopping [23] where the conductivity between two particles is highly dependent on particle spacing.

DES are called "switches" because when stretched they can switch the voltage applied to a DEA on or off. In turn DEA can deform switches-closing the loop and allowing for the creation of arbitrary electric circuits. Artificial muscle NAND gates [20], memory elements [24], and oscillators [20] have been built with the technology (and are pictured and explained in Fig. 3), DES controlled generators [25], motors [26] and ring oscillators [27] have also been built.

These artificial muscle circuits can be embedded into smart actuator networks to provide the distributed sensing and reflex-like-intelligence that is our goal. The problem however is that all of the demonstrated examples have used hand-painted carbon grease electrodes which suffer from rapid degradation over time, poor repeatability, long production time, poor scalability and an inability to miniaturize. Table 1 summarizes these disadvantages alongside the advantages of the technology.

If dielectric elastomer switches are to realize their potential, we need to address their limitations and develop more reliable materials and fabrication techniques. These materials should undergo large changes in resistivity when stretched, be tolerant of high electric fields, be miniaturizable, and tunable to different resistances. To put these requirements into perspective consider the NAND gate of Fig. 3. When reliably operated, DEA are limited to a roughly $10 \%$ strain, run at around $2500 \mathrm{~V}$, and draw on the order of $100 \mu \mathrm{A}$. Given these parameters the ideal piezo-resistor for the gate should not stiffen the membrane, should change with $10 \%$ compression from more than $2.5 \mathrm{GOhm}(1 \mu \mathrm{A}$ leakage) to less than $25 \mathrm{MOhm}$, and should be able to do this over many cycles.

One candidate technology to meet these goals is Filtered Cathodic Vacuum Arc (FCVA) gold ion implantation. In FCVA metal ion implantation, metal plasma is created by a high voltage discharge in a vacuum (Fig. 4) [28]. The content of the beam is filtered to remove big, heavy macro particles using a curved magnetic field—leaving only gold ions which are electrostatically accelerated to several $\mathrm{keV}$ to embed in a sample. Implanted electrodes consist of nanometer sized clusters of gold embedded up to $50 \mathrm{~nm}$ deep in a polydimethylsiloxane (PDMS) silicone matrix. If sufficiently small the gold clusters make electrical contact without mechanically bonding, creating a conductive and 

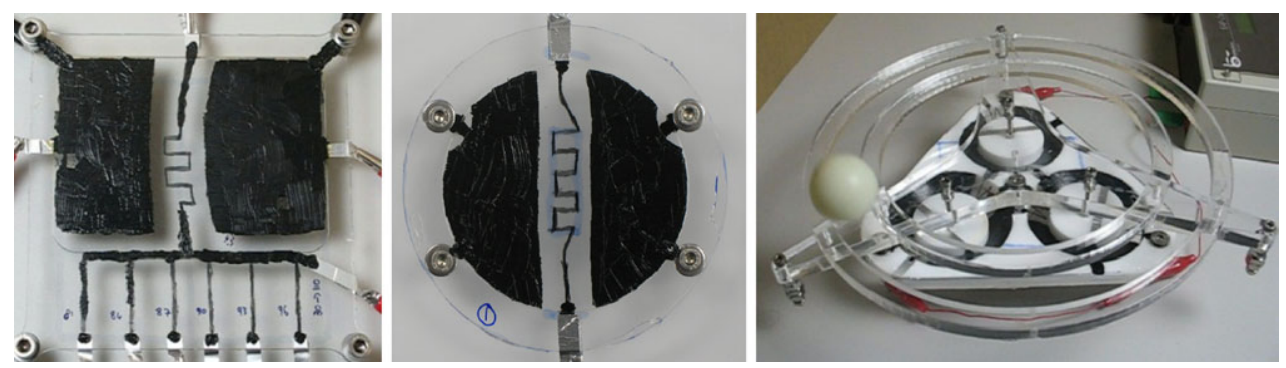

Fig. 3 On the left is an artificial muscle NAND gate [20]. Current through the central piezo-resistor is controlled via expansion of the two muscles. With combinations of NAND gates any other Boolean logic circuit can be built. In the middle is part of a memory element (or ring oscillator) $[24,27]$. By putting piezo-resistor/artificial muscle pairs into a state of positive feedback, volatile memory elements can be made. On the right is an oscillator [20] that made use of piezo-resistive switches to control an array of artificial muscles to roll a ball around some rails (Images reused from Refs. [20,27])

Table 1 Advantages and disadvantages of DES and conventional technology when applied to control networks of DEA

\begin{tabular}{|c|c|c|}
\hline & Advantages & Disadvantages \\
\hline Conventional electronics & Fast, precise, and mature technology. & $\begin{array}{l}\text { Rigid, poorly suited to high voltages }(>1 \mathrm{kV}) \text {, } \\
\text { incompatible manufacturing processes, large } \\
\text { number of interconnects, lack of soft sensors. }\end{array}$ \\
\hline Dielectric elastomer switches & $\begin{array}{l}\text { Cheap materials, fabrication methods same as DEA, } \\
\text { high voltage, soft and lightweight, inherently } \\
\text { coupled to devices, strain sensing functionality. }\end{array}$ & $\begin{array}{l}\text { Current materials do not last long, hand fabricated, } \\
\text { not miniaturizable, less precise, speed of sound } \\
\text { limited. }\end{array}$ \\
\hline
\end{tabular}

Fig. 4 FCVA ion implanter. $O n$ the left it can be seen that gold ions are accelerated from a plasma to the sample through a curved filter. On the right is a picture of the implanter in operation (Images reused from Niklaus [28])

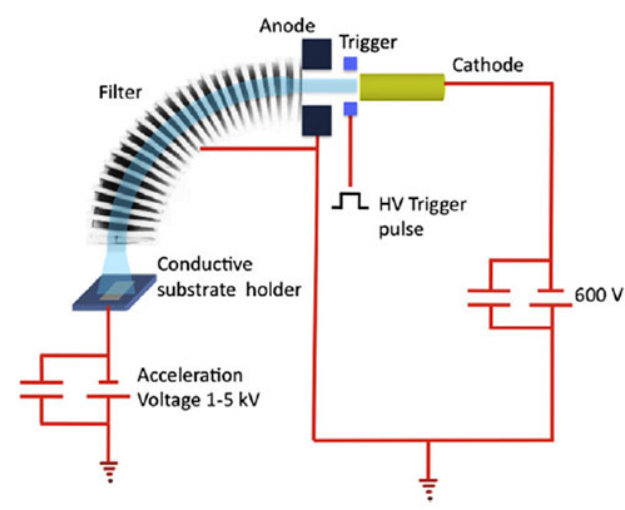

a

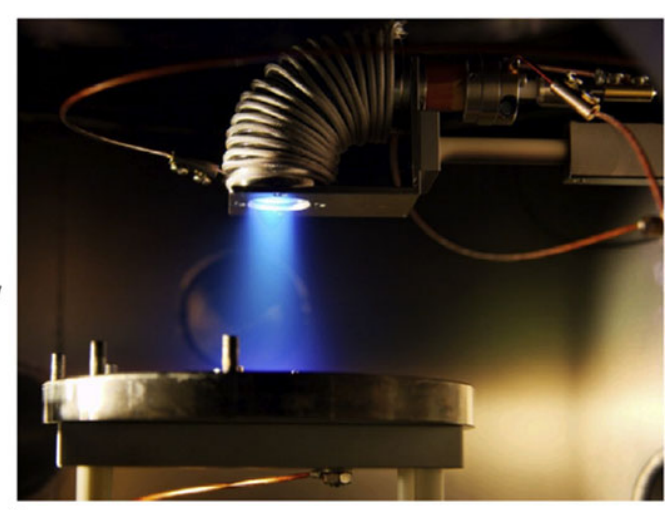

b stretchable electrode. FCVA gold ion implantation has been used to make stretchable electrodes for DEA in a range of applications including steerable mirrors, living cell stretchers, and microfluidic devices [28-32].

In this paper we use FCVA ion implantation to make implanted gold piezo-resistive electrodes. We first present a conceptual framework for designing ion implanted electrodes, then build and test piezo-resistors implanted to different densities of gold. We take the most promising category of resistor and apply it to make an artificial muscle inverter, and we finish by discussing the performance of the technology and future directions.

\section{Switching regimes}

Before we can make implanted gold piezo-resistors we need to understand the FCVA implanter. The implanter beam is pulsed and rastered over a sample to create a uniform distribution of ions. Many factors can influence implantation: source temperature, geometry, and alignment, chamber pressure, and electric field around the sample. These effects all contribute to varying dose per pulse or varying shape between pulses and are difficult to control. The best approach is to hold as many parameters constant between jobs, record data to establish trends, raster in a pattern to distribute any change in the dose per pulse across the device and control 
Fig. 5 (a) Schematic showing resistivity dose relationship with four distinct characteristic regions marked. (b) Actual dose-conductivity data reused from Niklaus [28] showing a strong percolation threshold

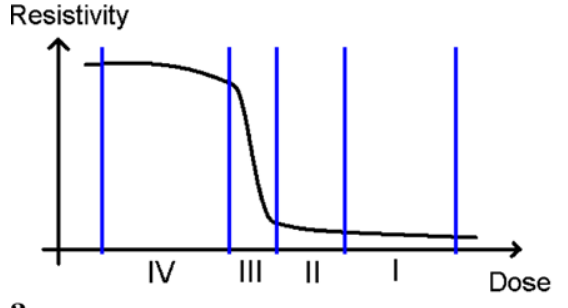

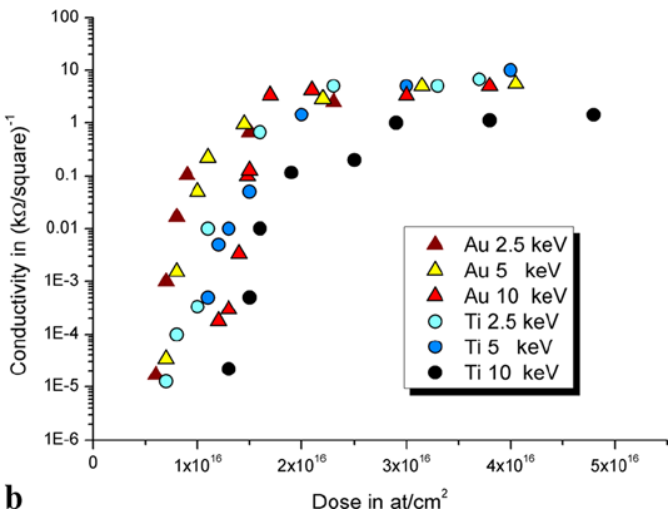

the process with a single parameter. The control parameter we chose was pulses $/ \mathrm{cm}^{2}$, which is the number of times the implanter fires a packet of gold ions at the substrate for a given area. We chose this parameter because we were aiming to control the quantity or "dose" of gold embedded in the PDMS, and the relationship between pulses $/ \mathrm{cm}^{2}$ and dose is the most repeatable and controllable.

Dose affects not only the initial resistivity of the electrodes, but also their piezo-resistive and mechanical properties. We can explain the resistivity-dose relationship by looking at the schematic in Fig. 5, which shows that for low doses the resistivity is very high, approaching that of the native PDMS. As the dose increases, the resistivity undergoes a sharp decrease as the gold particles percolate (form unbroken chains of particles from one side to the other), and from this point onwards there is a small increase in conductivity with dose. Crucially, as shown by Niklaus [28], the doses required for reaching electrical percolation are lower than those required for mechanical percolation: it is therefore possible to choose a dose where the electrical properties are a strong function of strain, but the mechanical properties will be mostly dominated by the PDMS substrate. As mentioned earlier, the starting resistance is only part of the picture-we propose that one can split the curve into four characteristic regimes:

I. High dose: In this regime, the gold has electrically and mechanically percolated, meaning that any resistors will be highly conductive and will crack easily. This region is useless for piezo-resistors that need to survive more than one cycle.

II. Above percolation: In this regime, the gold has percolated electrically, but not mechanically. The resistivity is very low so long and narrow electrodes need to be made.

III. On percolation: In this regime, the gold is in the process of percolating electrically. This enables to make electrodes of suitable resistance easily from a masking point of view. Unfortunately, this regime requires excellent dose control due to the highly sensitive nature of percolation phenomenon to dose near the percolation threshold (Fig. 5b).
IV. Low dose: In this regime, the gold has not percolated electrically. Resistors made in this regime must be very short and wide in order to have low enough resistivity.

\section{Materials and methods}

To test different implantation doses and geometries, a range of electrodes were made using the same fabrication process, as shown in Fig. 6. First, 25- $\mu \mathrm{m}$ thick PDMS (Dow Corning Sylgard 186) membranes were made using a Zenhtner ZUA2000 universal applicator and ZAA2300 automatic film coater on a glass plate coated with a watersoluble sacrificial layer. The membranes were cured at $80^{\circ} \mathrm{C}$ for 30 minutes, released from the glass plate after dissolution of the sacrificial layer, and transferred onto polyoxymethylene (POM) frames. The membranes were then pre-stretched equibiaxially to a factor of 1.25 in each direction and implanted using a $100-\mu \mathrm{m}$ thick stainless steel shadow mask to achieve the correct pattern. Once a membrane was implanted it was bolted in a sandwich between a POM and single-sided copper-coated epoxy frame. Interconnects in the copper-epoxy frame were filled with carbon grease (NYOGEL 786G) and copper tracks connected these to the bolt holes on the perimeter of the frame. To complete the device a central coupling was connected with Kapton tape (LewVac LLP, A-KTS-DS-25.4).

As shown in Fig. 7, resistance-stretch relationships were tested by displacing coupling points out of plane and measuring resistance at high voltage. Displacement was achieved with a Thorlabs manual precision stage. Resistance was measured at high voltage (powered with a Biomimetics Lab EAP controller) with a voltage divider and Fluke multimeter connected through a high-input impedance (Rimp). The sensor input impedance as well as supply voltage were changed to suit the piezo-resistor under study.

\section{Experimental results}

Figure 8 shows a high dose piezo-resistor (regime I) implanted at 75 pulses $/ \mathrm{cm}^{2}$. The resistor stretched until a crack 

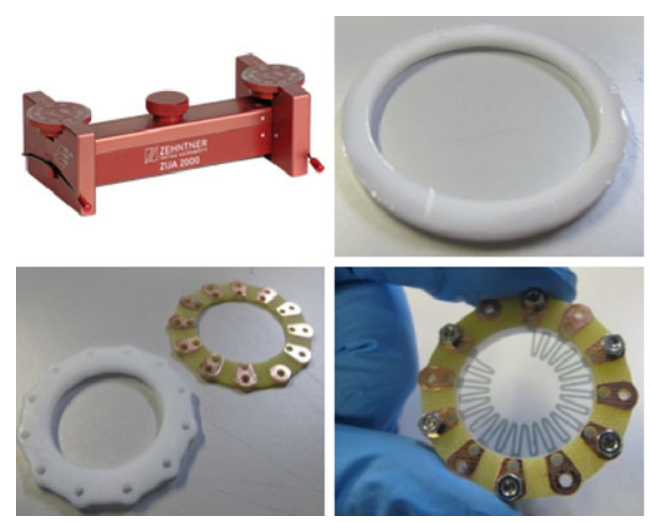

Fig. 6 Fabrication process for ion implanted piezo-resistors. From left to right, top to bottom: 1 . Silicone membranes are formed with a Zehntner universal applicator. 2. The silicone films were transferred onto frames. 3. The films were equibiaxially pre-stretched with an iris mechanism. 4. The films were masked and put into the implanter.
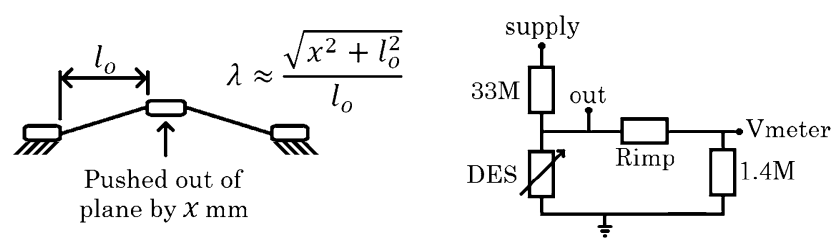

Fig. 7 To measure the resistance, the central coupling point was pushed out of plane. The approximate stretch ratio, $\lambda$, was found by Pythagoras' theorem where $x$ is the displacement out of plane and $l_{o}$ is the initial distance between the coupling point and the frame. To find the resistance, the circuit on the right of the figure was used

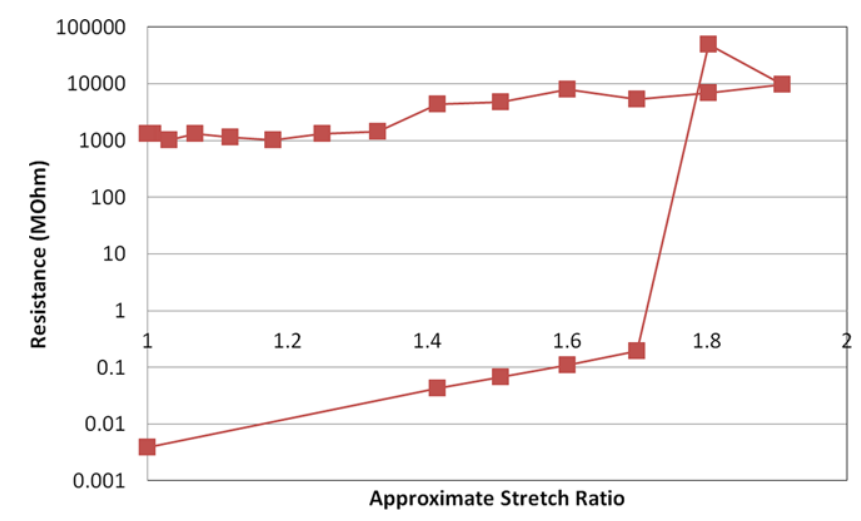

Fig. 8 Regime I switch. The piezo-resistor stopped conducting and broke down terminally after a single stretch. $2000 \mathrm{~V}$ supply, $100 \mathrm{MOhm}$ impedance, $75 \mathrm{pulses} / \mathrm{cm}^{2}$

formed in the gold electrode and then ablated so that it became permanently insulating. All regime I switches that were made exhibited this problem, heavily implanted electrodes became virtually impossible to handle as they would crack under the smallest strain. This cracking behavior is also exhibited by sputtered gold or silver electrodes and has been noted by others [33].

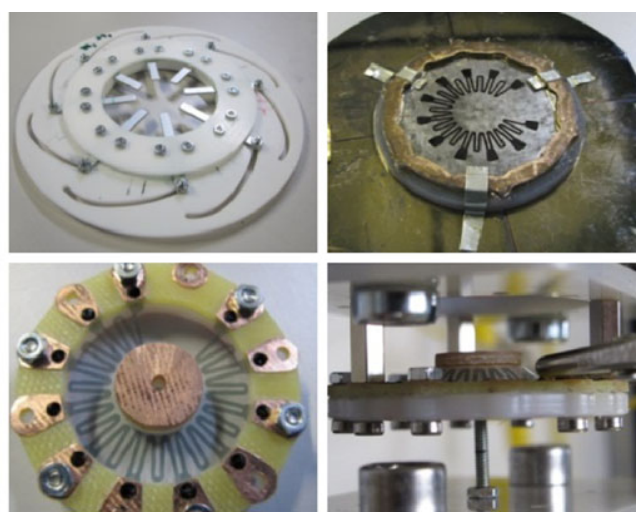

5. Frames were cut on a CNC router. 6. The implanted membrane was sandwiched between the frames. 7. Central coupling points were adhered, and carbon grease was used to connect. 8. The piezo-resistor was stretched and data collected

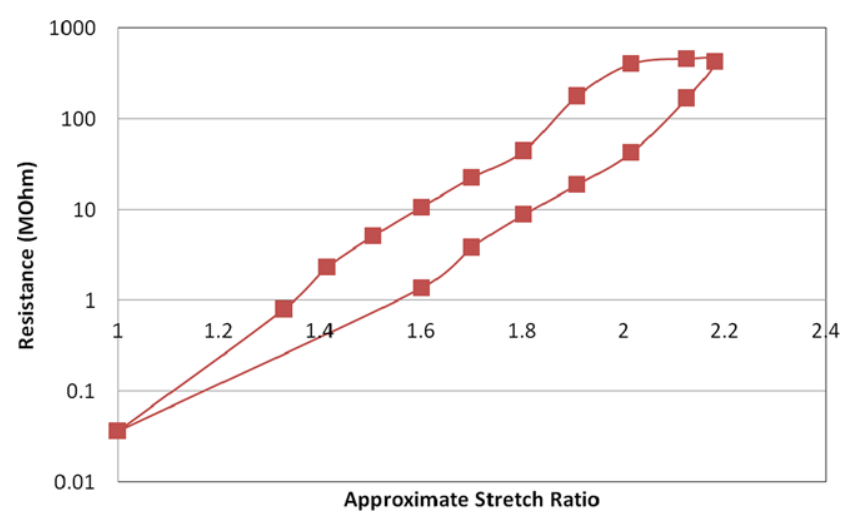

Fig. 9 Regime II switch. This type of electrode successfully switched high voltages when stretched, however suffered from hysteresis. $3500 \mathrm{~V}$ supply, $1 \mathrm{GOhm}$ impedance, 53 pulses $/ \mathrm{cm}^{2}$

Figure 9 shows stretch resistance data for a medium dose (regime II) piezo-resistor implanted at 53 pulses $/ \mathrm{cm}^{2}$. In comparison with the regime I switch of Fig. 8 the regime II switch had a slightly higher initial resistance, was capable of much larger strains, and returned to a conducting state when the stretch was released. To our knowledge this is the first reported use of a gold ion implanted piezo-resistor to switch high voltages. While this is promising, there are some problems: firstly, the device exhibits high hysteresis with nearly $20 \%$ dead stroke which is far outside of the reliable range of dielectric elastomer actuators; secondly, the response was not stable over time and decayed noticeably after the first few cycles as shown in Fig. 10 which is data for a similar regime II piezo-resistor.

It was not possible to reliably make regime III switches as the ion implanter process control was insufficient. Figure 11 shows the stretch resistivity curve for a regime IV piezo-resistor implanted at 52 pulses $/ \mathrm{cm}^{2}$ that increased in resistance over time: suggested to be the result of gold clus- 
ter conglomeration [30]. The piezo-resistor was stretched less than other devices due to limits in the resistance sensor setup. Note that this piezo-resistor has roughly the same pulse density as the regime II switches of Figs. 9 and 11, highlighting the large influence that other process parameters can have on electrode behavior.

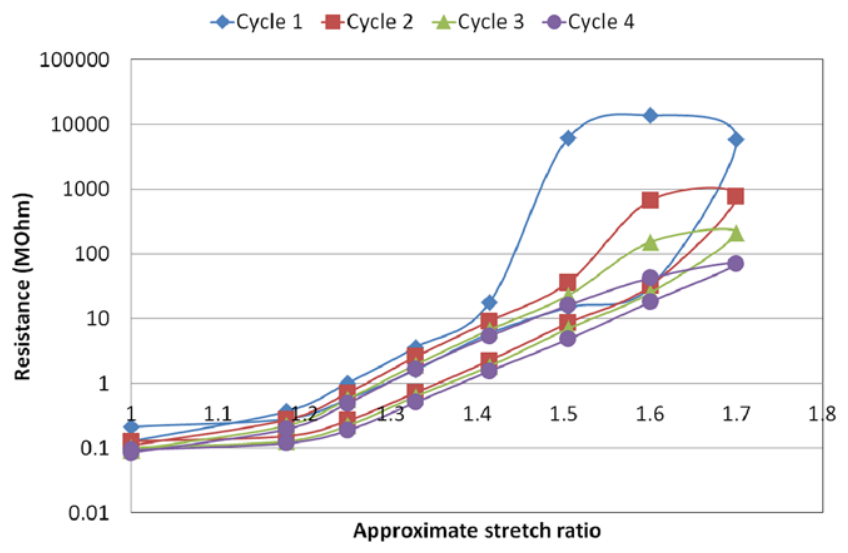

Fig. 10 Regime II switches degraded with stretching cycles. $3000 \mathrm{~V}$ supply, $1 \mathrm{GOhm}$ impedance, $51 \mathrm{pulses} / \mathrm{cm}^{2}$

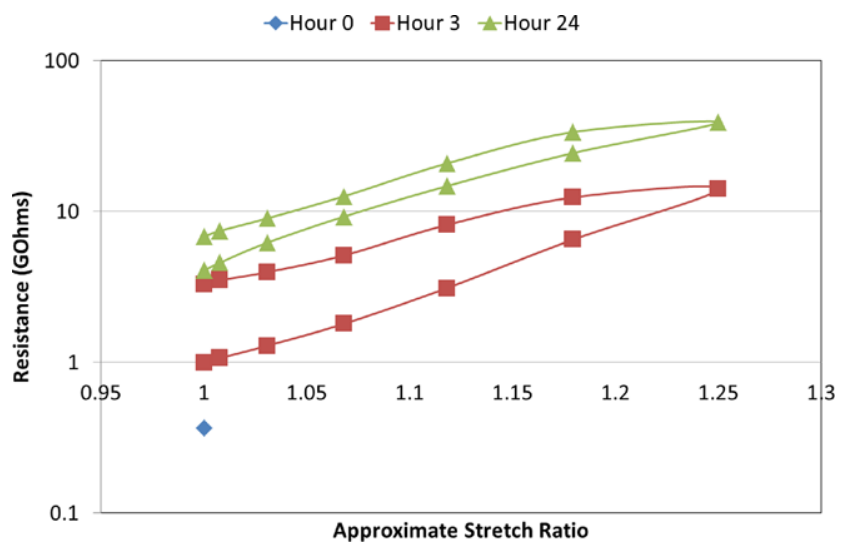

Fig. 11 Resistance vs. stretch for a regime IV switch at high voltage. The piezo-resistor was able to change in resistance by nearly an order of magnitude with stretch; however, it aged rapidly with its resistance increasing substantially in a 24-hour period. $3000 \mathrm{~V}$ supply, $1 \mathrm{GOhm}$ impedance, 52 pulses $/ \mathrm{cm}^{2}$

\section{The inverter}

The regime II switches showed promise for creation of artificial muscle circuits, so they were used to create an artificial muscle inverter. Inverters are important elements in logic with high output when the input is low, and with low output when the input is high. They can form ring oscillators which can be used directly as drivers in artificial muscle devices_-such as microfluidic pumps_-and provide a good benchmark component to test new materials against [27]. To make the inverter an actuator was coupled to a regime II switch (64 pulses $/ \mathrm{cm}^{2}$ ) as shown in Fig. 12. The central coupling point was attached to a weight that displaced it downwards. When the DEA was actuated, the piezo-resistor would stretch causing it to become non-conductive. The piezo-resistor was configured into a voltage divider circuit with a 100-MOhm discharge resistor so that when a high voltage was applied to the DEA, the output would drop low. The output was measured using a 1-GOhm input impedance voltage divider coupled to a fluke multimeter.

The inverter inverted the input signal with a gain of -0.14 (change in output voltage divided by change in input voltage) as shown in Fig. 13. The low gain is due to the high hysteresis and low sensitivity of category 2 switches coupled with the small actuation of DEA. It could be improved by using bigger actuators with better process control so that all the sections would switch simultaneously or by utilizing higher supply voltage than actuator driving voltage. Nevertheless, this is the first circuit element made using ion implanted DES.

\section{Discussion}

We have demonstrated the use of ion implanted gold electrodes as high voltage piezo-resistive switches, DES, and created a gold ion implanted artificial muscle inverter. While the piezo-resistors and inverter presented in this paper are not developed to the point where they can be directly applied to make generic smart artificial muscle networks, we believe that they show strong promise as they are both applications for, and clear roads of improvement to, devices formed out of two of the four regimes.
Fig. 12 Inverter. From left to right: (a) When the DEA (top) actuated, the DES (bottom) was stretched and the mass lowered. (b) The piezo-resistor was configured in a circuit to invert the signal to the DEA. (c) A photo of the fabricated inverter with the actuator membrane (top) and the switch membrane (bottom)

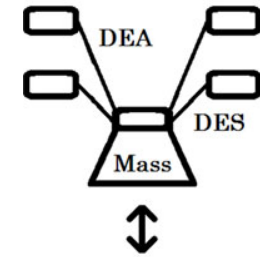

a

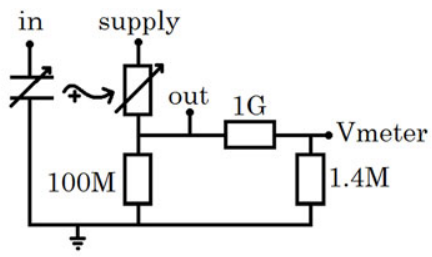

b

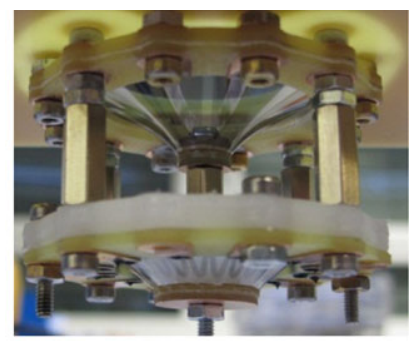

c 


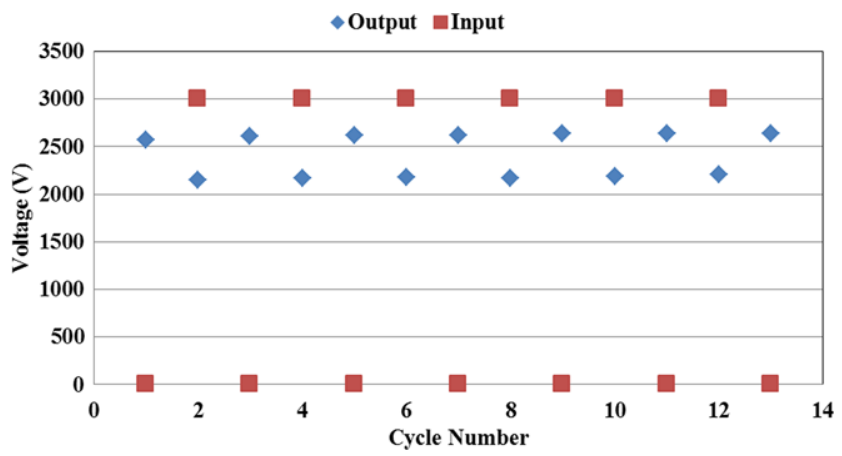

Fig. 13 Category 2 inverter with a 64 pulses $/ \mathrm{cm}^{2}$ switch. The input was turned on and off at $0.1 \mathrm{~Hz}$ and the output recorded

Regime II switches have high hysteresis, outside of the range that silicone DEA can be reasonably hoped to overcome as shown by the inverter. However, there are ways around this approach, such as the use of mechanical amplification to increase the strain on the piezo-resistor relative to the DEA or the use of higher strain DEA technologies that are under development. Another issue with regime II switches is their relatively low resistivity, this can be overcome with the creation of long and winding tracks using photolithography process, or by developing higher current (and speed) circuits such as might be needed for power generation. Indeed, power generation might be an ideal application for these piezo-resistors as the high hysteresis may prevent losses during switching, stretches on the order of $100 \%$ are common, and low resistivities increase efficiency. There are other applications for high hysteresis switches such as hysteretic oscillators or debouncing circuits.

Regime III switches are currently very difficult to realize with existing process control. Fortunately, there are clear roads to improvement such as direct inline dose control or indirect process control of source temperature or geometry. High-dose regime I switches crack and burn out permanently after being stretched, which makes them unsuitable for the creation of smart actuator networks. Low-dose regime IV switches are unstable and degrade over time, so at this point we see also little use for these devices.

There has been a strong trend in recent decades away from mechanistic control and towards the use of discrete sensors and microprocessors to provide feedback in mechatronic systems. In the face of these advances the dielectric elastomer switch approach comes full circle and makes use of mechanistic approach. There are some fundamental differences, however: DES are soft, they are directly and intimately coupled to artificial muscles, and they can draw on design and control concepts from the digital revolution. While the devices presented here were of centimeter scale, the ion implantation technology is fundamentally miniaturizable. Once in-plane architectures (that are also miniaturizable) are developed, artificial muscle circuits should enjoy advantages such as greater complexity and higher speed.
This last point is a final major distinction from the past. The mechanical coupling of artificial muscles is limited by the speed of sound in the material, so that as devices get shrunk the possibility of bandwidths in the $\mathrm{kHz}$ range or beyond could become a reality.

\section{Conclusions}

In this preliminary study we have demonstrated that gold ion implanted piezo-resistive electrodes can switch the high voltages required for artificial muscles. We showed that there are different regimes of behavior in which implanted piezo-resistors can be chosen to operate, and built an artificial muscle inverter using this technology. Future work involves exploring different metal ions, improving implanter process control and testing applications such as energy harvesting or hysteretic oscillators.

While implanted piezo-resistors currently show too much hysteresis to be used in the creation of the arbitrary artificial muscle circuitry required to imitate the success of smart muscular networks in nature, the fabrication process and preliminary experiments show substantial promise. Ion implanted circuitry can be miniaturized, patterned, and integrated directly into artificial muscle networks. Once challenges in process control and material hysteresis are overcome, we will be closer to a future where nature has a monopoly on soft smart actuator networks no more.

Acknowledgements This work was partially funded by the Royal Society of New Zealand via B.M. O'Brien's Rutherford Foundation Postdoctoral Fellowship, the Swiss National Science Foundation grant 200020-130453, the Indo Swiss Joint Research Programme (ISJRP) and the Auckland Bioengineering Institute. The authors would like to thank L. Maffli, S. Akbari, P. Rinne, M. Poliero, V. Perret, P. Rosset, S. Pilkington, and M. O'Brien.

\section{References}

1. D. Trivedi, C.D. Rahn, W.M. Kier, I.D. Walker, Soft robotics: biological inspiration, state of the art, and future research. Appl. Bionics Biomech. 5(3), 99-117 (2008)

2. R.E. Pelrine, R.D. Kornbluh, Q. Pei, J.P. Joseph, High-speed electrically actuated elastomers with strain greater than $100 \%$. Science 287, 836-839 (2000)

3. I.A. Anderson, T.A. Gisby, T.G. McKay, B.M. O’Brien, E.P. Calius, Multi-functional dielectric elastomer artificial muscles for soft and smart machines. J. Appl. Phys. 112(4), 041101 (2012)

4. R.E. Pelrine, R.D. Kornbluh, Q. Pei, S. Stanford, S. Oh, J. Eckerle, R. Full, M. Rosenthal, K. Meijer, Dielectric elastomer artificial muscle actuators: toward biomimetic motion. Proc. SPIE 4695 (2002)

5. K. Jung, J.C. Koo, J.-d. Nam, Y.K. Lee, H.R. Choi, Artificial annelid robot driven by soft actuators. Bioinspir. Biomim. 2, S42S49 (2007)

6. B. O'Brien, T. Gisby, S. Xie, E. Calius, I. Anderson, FEA of dielectric elastomer minimum energy structures as a tool for biomimetic design. Proc. SPIE 7287 (2009) 
7. C. Jordi, S. Michel, E. Fink, Fish-like propulsion of an airship with planar membrane dielectric elastomer actuators. Bioinspir. Biomim. 5(2) (2010)

8. Q. Pei, M.A. Rosenthal, R. Pelrine, S. Stanford, R.D. Kornbluh, Multifunctional electroelastomer roll actuators and their application for biomimetic walking robots. Proc. SPIE 5051 (2003)

9. J. Rossiter, P. Walters, B. Stoimenov, Printing 3D dielectric elastomer actuators for soft robotics. Proc. SPIE 7287 (2009)

10. B.A. Trimmer, New challenges in biorobotics: incorporating soft tissue into control systems. Appl. Bionics Biomech. 5(3), 119-126 (2008)

11. G. Sumbre, Y. Gutfreund, G. Fiorito, T. Flash, B. Hochner, Control of octopus arm extension by a peripheral motor program. Science 293(5536), 1845-1848 (2001)

12. Y. Yekutieli, G. Sumbre, T. Flash, B. Hochner, How to move with no rigid skeleton? Biologist 49(6), 250-254 (2002)

13. T.A. Gisby, S. Xie, E.P. Calius, I.A. Anderson, Integrated sensing and actuation of muscle-like actuators. Proc. SPIE 7287 (2009)

14. M. Migita, E. Mizukami, Y.P. Gunji, Flexibility in starfish behavior by multi-layered mechanism of self-organization. Biosystems 82(2), 107-115 (2005)

15. K. Agata, T. Inoue, Survey of the differences between regenerative and non-regenerative animals. Dev. Growth Differ. 54(2), 143-152 (2012)

16. T. Rubilar, C. Pastor, E. Díaz de Vivar, Timing of fission in the starfish Allostichaster capensis (Echinodermata: Asteroidea) in laboratory. Rev. Biol. Trop. 53(Suppl 3), 299-303 (2005)

17. J.A. Rogers, Y. Huang, A curvy, stretchy future for electronics. Proc. Natl. Acad. Sci. USA 106(27), 10875-10876 (2009)

18. D.H. Kim, N. Lu, R. Ma, Y.S. Kim, R.H. Kim, S. Wang, J. Wu, S.M. Won, H. Tao, A. Islam, K.J. Yu, T.I. Kim, R. Chowdhury, M. Ying, L. Xu, M. Li, H.J. Chung, H. Keum, M. McCormick, P. Liu, Y.W. Zhang, F.G. Omenetto, Y. Huang, T. Coleman, J.A. Rogers, Epidermal electronics. Science 333(6044), 838-843 (2011)

19. A. Jong-Hyun, J. Jung Ho, Stretchable electronics: materials, architectures and integrations. J. Phys. D, Appl. Phys. 45(10), 103001 (2012)

20. B.M. O'Brien, E.P. Calius, T. Inamura, S.Q. Xie, I.A. Anderson, Dielectric elastomer switches for smart artificial muscles. Appl. Phys. A, Mater. Sci. Process. 100(2), 385-389 (2010)
21. L. Chen, G. Chen, L. Lu, Piezoresistive behavior study on fingersensing silicone rubber/graphite nanosheet nanocomposites. Adv. Funct. Mater. 17(6), 898-904 (2007)

22. G. Canavese, M. Lombardi, S. Stassi, C.F. Pirri, Comprehensive characterization of large piezoresistive variation of Ni-PDMS composites. Appl. Mech. Mater. 110-116, 1336-1344 (2012)

23. R. Strümpler, J. Glatz-Reichenbach, Conducting polymer composites. J. Electroceram. 3(4), 329-346 (1999)

24. B.M. O'Brien, T.G. McKay, S.Q. Xie, E.P. Calius, I.A. Anderson, Dielectric elastomer memory. Proc. SPIE 7976 (2011)

25. T.G. McKay, B.M. O'Brien, E.P. Calius, I.A. Anderson, Soft generators using dielectric elastomers. Appl. Phys. Lett. 98, 142903 (2011)

26. B.M. O’Brien, T.G. McKay, T.A. Gisby, I.A. Anderson, Rotating turkeys and self-commutating artificial muscle rotary motors. Appl. Phys. Lett. 100, 074108 (2012)

27. B.M. O'Brien, I.A. Anderson, An artificial muscle ring oscillator. IEEE/ASME Trans. Mechatron. 17(1), 197-200 (2012)

28. M. Niklaus, H.R. Shea, Electrical conductivity and Young's modulus of flexible nanocomposites made by metal-ion implantation of polydimethylsiloxane: the relationship between nanostructure and macroscopic properties. Acta Mater. 59(2), 830-840 (2011)

29. S. Rosset, M. Niklaus, P. Dubois, H.R. Shea, Large-stroke dielectric elastomer actuators with ion-implanted electrodes. J. Microelectromech. Syst. 18(6), 1300-1308 (2009)

30. S. Rosset, M. Niklaus, P. Dubois, H.R. Shea, Metal ion implantation for the fabrication of stretchable electrodes on elastomers. Adv. Funct. Mater. 19(3), 470-478 (2009)

31. P. Dubois, S. Rosset, S. Koster, J. Stauffer, S. Mikhailov, M. Dadras, N.-F. deRooij, H. Shea, Microactuators based on ion implanted dielectric electroactive polymer (EAP) membranes. Sensors Actuators A 130-131, 147-154 (2006)

32. S. Akbari, H.R. Shea, Microfabrication and characterization of an array of dielectric elastomer actuators generating uniaxial strain to stretch individual cells. J. Micromech. Microeng. 22(4) (2012)

33. R.E. Pelrine, R.D. Kornbluh, J.P. Joseph, R. Heydt, Q. Pei, S. Chiba, High-field deformation of elastomeric dielectrics for actuators. Mater. Sci. Eng. C 11, 89-100 (2000) 